This item was submitted to Loughborough's Research Repository by the author.

Items in Figshare are protected by copyright, with all rights reserved, unless otherwise indicated.

\title{
Improving water supply and sanitation programme effectiveness: lessons from WaterAid's outcome evaluation studies
}

PLEASE CITE THE PUBLISHED VERSION

http://dx.doi.org/10.1111/j.1747-6593.2012.00317.x

\section{PUBLISHER}

John Wiley \& Sons, Inc .๑ The Authors. Water and Environment Journal @ CIWEM

VERSION

AM (Accepted Manuscript)

LICENCE

CC BY-NC-ND 4.0

\section{REPOSITORY RECORD}

Cotton, Andrew P., Jerry Adams, and David Shaw. 2019. "Improving Water Supply and Sanitation Programme Effectiveness: Lessons from Wateraid's Outcome Evaluation Studies”. figshare.

https://hdl.handle.net/2134/11490. 
This item was submitted to Loughborough's Institutional Repository (https://dspace.lboro.ac.uk/) by the author and is made available under the following Creative Commons Licence conditions.

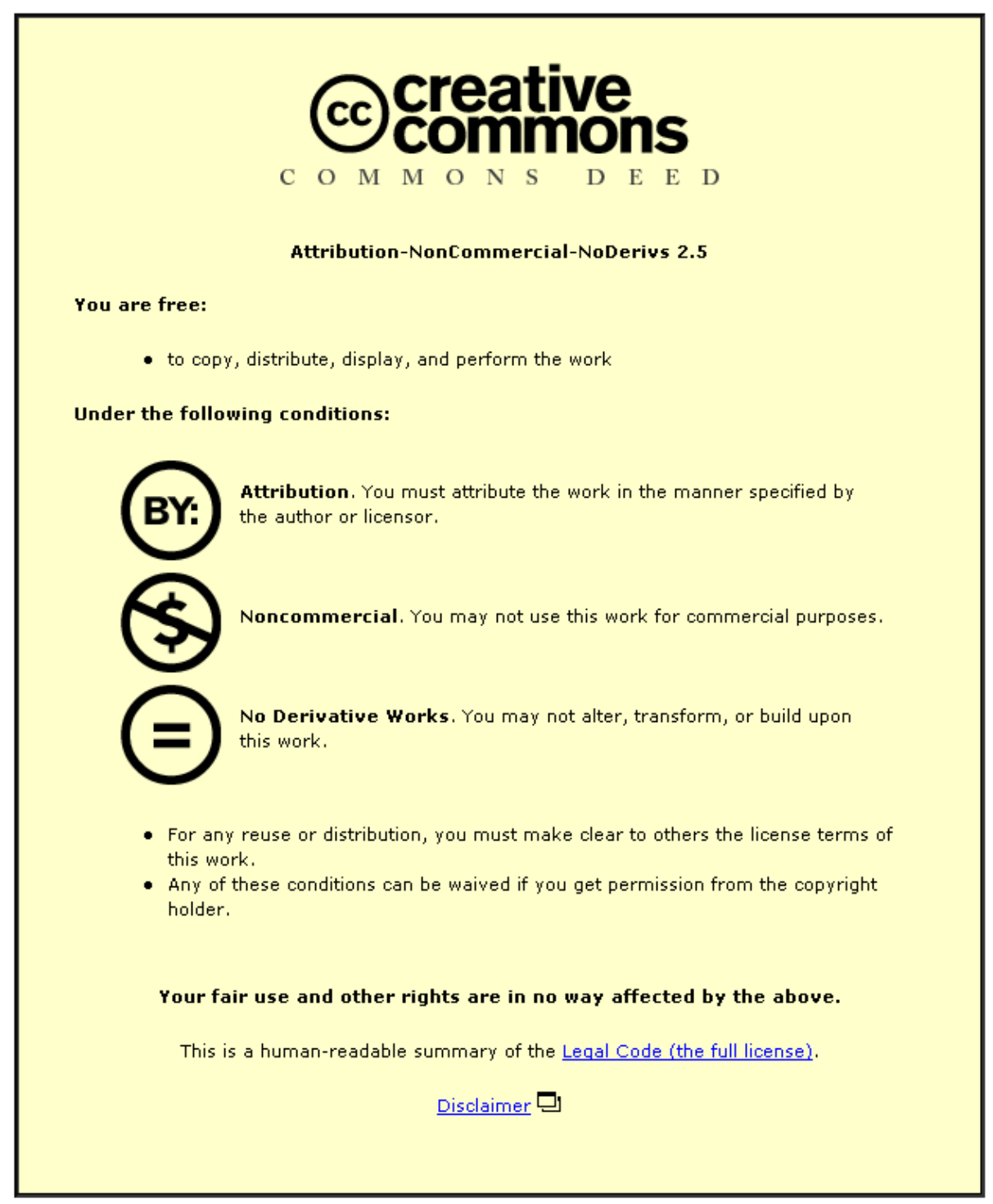

For the full text of this licence, please go to: http://creativecommons.org/licenses/by-nc-nd/2.5/ 


\title{
Improving Water Supply and Sanitation Programme Effectiveness: Lessons from WaterAid's Outcome Evaluation Studies
}

\author{
Andrew Cotton ${ }^{1}$, Jerry Adams ${ }^{2} \&$ David Shaw ${ }^{2}$ \\ ${ }^{1}$ WEDC, Loughborough University, Loughborough, UK and ${ }^{2} \mathrm{PME}$, WaterAid, London, UK
}

\begin{abstract}
Many low income countries are off-track to reach the Millennium Development Goals for water supply and sanitation. This paper develops a theoretical framework and methodology to improve the evaluation of water and sanitation programme outcomes, focusing on relevance, effectiveness and sustainability. The resulting set of evaluation questions are applied to seven independent evaluations of WaterAid's country programmes. Results are synthesised by assigning an ordinal ranking to the findings which are aggregated to assess overall outcome status that has validity at an organisational level, rather than just at country programme level. Strong areas of performance are partnership development, policy advocacy, equity and inclusion. Areas requiring attention are organisational learning, programme structure and government partner capacity for sustainability. The findings will be used to prioritise future programme management and are clustered into seven outcome areas that are of generic interest and significance to other organisations managing water supply and sanitation programmes.
\end{abstract}

\section{Keywords}

health; impact; local government; sustainability; waste; water supply.

\section{Introduction}

The United Nations Development Summit in 2000 agreed a set of time-bound and measurable goals for international development efforts. Target 7c of the Millennium Development Goals (MDGs) is "to halve, by 2015, the proportion of people without sustainable access to safe drinking-water and basic sanitation". With 2015 rapidly approaching, the world is generally ontrack to reach the drinking water target whilst access to sanitation is the most off-track of all the MDG targets. Globally, 884 million people are without improved sources of drinking water and 2.6 billion people do not use improved sanitation (JMP 2010).

These global averages mask important differences, with many low-income and lower-middle income countries facing major challenges in providing even the most basic levels of service. For example, $37 \%$ of those unserved by safe drinking water live in Sub-Saharan Africa and $72 \%$ of those without access to improved sanitation live in Asia (JMP 2010). Furthermore, the Global Annual Assessment of Sanitation and Drinking Water report (GLAAS 2010) presents a bleak picture of the financial resources flowing to the sector with 35 out of the 37 countries surveyed reporting that financial flows were insufficient to achieve the MDG target for sanitation and 32 having insufficient to reach the drinking water target. In 2008, development aid for sanitation and drinking water amounted to US\$7.4 billion (GLAAS 2010) whereas global cost estimates to reach the MDG target vary enormously from US $\$ 6.7$ billion to US $\$ 75$ billion per year depending on the assumptions made (WELL 2005).

WaterAid is an international non-governmental organization with a mission to transform lives by improving access to safe water, hygiene and sanitation in the world's poorest communities. WaterAid works with partner organisations to implement projects in 26 countries in Africa, Asia and the Pacific region. Through advocacy work, WaterAid influences sector stakeholders on important policy issues at both a country and international level. During 2010/11, WaterAid invested $£ 32.9$ million (of a total $£ 50.8$ million) with partners to deliver water, sanitation and hygiene work, thereby supporting 1.5 million people to access safe water and 1.6 million people 
with access to sanitation. In total, since 1981, WaterAid have reached almost 16 million people with safe water and, since 2004, more than 11 million people with sanitation.

WaterAid is acutely conscious of the compelling need to accelerate the delivery of sustainable water supply, sanitation and hygiene (WASH) services, particularly for the poorest. Continuous performance improvement is essential in order to make most effective use of the limited resources available and WaterAid therefore undertakes regular evaluations of its Country Programmes. However, WaterAid's Programme Effectiveness Unit found that evaluation findings were very difficult to aggregate at the organisational level and that documented experience from the wider WASH sector was difficult to assimilate into improved programme design and management. This creates real difficulties for practitioners seeking operational guidance to improve their programmes in the search for genuinely sustainable water and sanitation solutions.

This paper addresses the problem of how to measure and improve programme effectiveness through the use of studies to evaluate the outcomes of water, sanitation and hygiene interventions. The paper develops the theoretical background underlying WaterAid's outcome evaluation policy and illustrates how its consistent application to seven country programme evaluation studies can be used for meta-analysis of the data. The aim is to identify lessons concerning programme effectiveness that are applicable at the organisational level. Ultimately, this approach has the potential to offer a more coherent view of collective experience in the water and sanitation sector, in order to avoid making the same mistakes and "reinventing the wheel".

\section{The problem of identifying 'what works'}

\section{Making sense of what we know}

Adams (2001) noted that performance evaluations carried out by NGOs often consisted only of descriptions of activities rather than analysis of programme relevance and effectiveness. In an effort to aggregate findings from different urban sanitation programmes, Saywell and Hunt (1999) noted "Documentation of the... lessons to be learned...is haphazard".

Possible contributing factors are firstly the wide range of interventions that can arise from the mix of technical, environmental, financial, institutional and social aspects that contribute to WASH programme development (WELL 1998); and secondly a lack of consistency and rigour in evaluation methodologies (Adams 2001). This leads to difficulties with attribution of factors that give rise to positive, negative or neutral outcomes.

Nevertheless, considerable effort goes into reporting past experiences and attempting to draw out lessons (WordPress undated, IRC 2011). However, meta-analysis of the data and synthesis of common themes is fraught with difficulty if only single cases are reported and there is lack of consistency in the evaluation questions used. The external validity of findings, that is the applicability of results to other situations, becomes open to question (Denscombe 2010). A further consequence is that the "lessons learned" may be able to offer little more than superficial statements of the self-evident.

\section{What are we trying to measure?}

A useful starting point is to ensure clarity about the point of measurement of an evaluation and of the data sets that are to be co-analysed. Adams (2001) offers the following explanation.

1. Inputs measure the use of financial and human resources over a particular period of time; these are usually assessed through regular programme monitoring (rather than evaluation).

2. Outputs measure effort, with indicators concerning the implementation of activities; an example would be the number of waterpoints constructed over a particular period of time. 
3. Outcome measures the effectiveness of programme interventions, with indicators measuring the use of outputs and sustained use or production of benefits.

4. Impact measures change with indicators that assess longer term life-changing aspects.

At one end of the spectrum, project and programme monitoring of inputs and outputs allows comparison of progress against what was scheduled at the outset and helps to guide future resource allocation to reach project targets. It does not give any indication as to whether the outputs are achieving the overall objectives of the programme.

At the other end of the spectrum, an important application of impact evaluation is quantifying the health effects of interventions in water supply, sanitation and hygiene through meta-analysis of randomised and non-randomised control trials. These evaluations follow an agreed approach for undertaking systematic reviews of data sets (Higgins and Green 2011). Such impact studies are crucial in providing high-level evidence to justify overall investments in water and sanitation (Clasen et al. 2010, Waddington et al. 2009).

In the middle of this spectrum, the distinction between outcome and impact is not always clear. In terms of a logic model, there can be multiple intermediate (short- and medium-term) outcomes over time that eventually lead to impact-some or all of which may be included in an evaluation of impact at a specific moment in time Thus outcome and impact can be viewed as stages along a results continuum (Leeuw and Vaessen 2009).

The difficulties experienced by WaterAid in distilling generically applicable lessons from experience are symptomatic of the "missing middle" of aggregated learning in the water and sanitation sector. It is at the level of outcome that the evidence necessary to improve the direction and focus of programmes to optimise scarce resources can be identified.

\section{Framework and Methodology for Outcome Evaluation}

Drawing valid lessons at the organisational level and more widely for the WASH sector as a whole requires meta-analysis of case studies that have a common point of measurement. Filling this gap - the "missing middle" - requires an outcome evaluation framework with a sound theoretical base that can be applied consistently both within an organisation and more widely by others active in WASH. The evaluation framework proposed by WaterAid is based on a theory of change (Leeuw and Vaessen 2009) that links interventions to outcomes coupled with rigorous, consistent outcome monitoring.

WaterAid's evaluation framework is grounded in the OECD Development Assistance Committee's (DAC) guidelines for evaluating development projects (Development Assistance Committee undated, 1991) which identifies five key attributes.

1. Relevance: "the extent to which the aid activity is suited to the priorities \& policies of the target group, recipient \& donor".

2. Effectiveness: "a measure of the extent to which an aid activity attains its objectives".

3. Efficiency: "this measures the outputs -- qualitative \& quantitative -- in relation to the inputs".

4. Impact: "the positive and negative changes produced by a development intervention, directly or indirectly, intended or unintended".

5. Sustainability: "this involves the main impacts and effects resulting from the activity on the local social, economic, environmental and other development indicators. The examination should be concerned with both intended and unintended results".

A single evaluation study will rarely address all five attributes; it is essential that evaluation studies are clear about their precise objectives. WaterAid's approach to outcome evaluation 
focuses specifically on: relevance, that is, the extent to which the Country Programme is aligned with the national context; and effectiveness, that is, the extent to which the Country Programme has addressed identified needs and priorities. Sustainability is incorporated as a component of effectiveness, with a specific focus on operation and maintenance of services.

WaterAid's Global Strategy (WaterAid 2009) sets out organisational aims and cross cutting principles; these articulate the theory of change through which WaterAid operates to achieve the change it seeks. There are four global aims.

1. Aim 1: to promote and secure poor people's rights and access to safe water, improved hygiene and sanitation

2. Aim 2: to support governments and service providers in developing their capacity to deliver safe water, improved hygiene and sanitation

3. Aim 3: to advocate for the essential role of safe water, improved hygiene and sanitation in human development.

4. Aim 4: to further develop as an effective global organisation recognised as a leader in our field and for living our values

These are supported by six cross-cutting principles that underpin WaterAid's way of working, namely: working in partnership; equity and inclusion; sustainability; accountability; learning; integration of water, sanitation and hygiene. These aims and principles are reflected in the multiyear plans and budgets of WaterAid's Country Programmes that specify outputs, activities and inputs.

WaterAid's policy for country programme evaluations (WaterAid 2010) sets out an evaluation framework linking this organisational theory of change with the DAC criteria. This leads to a set of 17 evaluation questions that are categorised into seven broad outcome areas, shown in Table 1. These are also likely to be of generic interest and significance to other organisations, both government and non-government, managing WASH programmes. This model is applied to the seven country programme evaluations in order to undertake meta-analysis of the findings.

\section{Data Collection and Analysis}

WaterAid's policy for country programme evaluations (WaterAid 2010) links this organisational theory of change to the DAC evaluation criteria. The policy articulates this link through a set of 17 standard evaluation questions that are categorised into seven broad outcome areas as shown in Table 1; these questions are applied to all country programme evaluation studies. At the country level, each programme develops a five-year strategy (based on the corporate global aims) with an associated performance framework and indicators that reflect the results to be achieved by the programme This performance framework provides the objective criteria against which the individual evaluation studies are carried out, using the standardised evaluation questions shown in Table 1.

Data collection is undertaken by an evaluation team (usually four). The main tools used for data collection are: document review and analysis including financial and output performance data; key informant interviews with government, bilateral and multilateral agencies, partner NGOs, Community-Based Organizations (CBOs) and service users at household and community level; field observation in different communities that make use of the different water and sanitation technologies. Care is taken to triangulate views of informants through consistent application of evaluation sub-questions, which are disaggregated and targeted by informant type.

The first stage of data analysis involves aggregation by the evaluation team of the findings from different data sources for each of the evaluation questions. Performance is assessed by 
comparing the findings (for each evaluation question) with the objective criteria set out in the performance framework for the country programme. Performance against each of these 17 evaluation questions is then assigned an ordinal score by the evaluation team as follows.

$\begin{array}{cl}\text { Evaluation Score } & \text { Description } \\ 0 & \text { Extremely unsatisfactory } \\ 1 & \text { Barely adequate, requiring major improvements } \\ 2 & \text { Satisfactory, but with much need for improvement } \\ 3 & \text { Good, but some areas for improvement identified } \\ 4 & \text { Very good, only minor improvements needed } \\ 5 & \text { Excellent, outstanding, exceptional }\end{array}$

The ordinal scale is used to indicate relative position (not the magnitude of the difference) in relation to the characteristics of performance (Malhotra and Birks 2000). The primary purpose and use of ordinal scoring in this work is to identify those programme areas that are relatively high performing or low performing. Furthermore, the approach clearly identifies those areas of the underpinning data that need to be explored in order to fully understand the issues that are determining performance. WaterAid's evaluation policy specifies six ordinal groupings as the minimum necessary to best suit the ultimate application of the evaluation findings in recommending future changes in country programme implementation and strategy. Adding to the number of ordinal points would be likely to lead to increased variability between the different country evaluations without providing significant additional clarity or specificity in terms of application of the evaluation findings to the future strategic direction of the programmes.

\section{Limitations on the Evaluation Data Set}

Data from evaluation studies was available from Malawi (2008), Zambia (2009), Ethiopia (2009), Nigeria (2009), Uganda (2010), Ghana (2010) and Mozambique (2011). Earlier studies were not included because WaterAid's current Global Strategy was not in place and earlier evaluations were therefore not necessarily aiming to assess country programmes against the framework outlined above. A difficulty with the current data set arises in that whilst the focus of evaluation studies was firmly at the level of outcomes (that is, relevance and effectiveness), the phrasing of specific evaluation questions adopted by evaluators varied prior to the implementation of WaterAid's revised evaluation policy. This means that it is not possible to do cross-comparisons between all countries for all evaluation questions.

\section{Results and Discussion}

Table 1 summarises the ordinal scoring for each evaluation question for the seven studies.

\section{Programme Effectiveness: Assigning Outcome Status}

In order to assess programme effectiveness at the organisational level it is necessary to attempt meta-analysis of the findings across the range of comparable country programme evaluation studies. This is approached by aggregating the findings for each evaluation question across the 7 
studies into an 'outcome status' using the ordinal scores developed above. The 'outcome status' for each of the 17 evaluation questions is defined as follows.

\begin{tabular}{|c|c|}
\hline Outcome Status & Basis \\
\hline Strong & $\begin{array}{l}\text { Three or more scores of } 4 \text { and no more than one score of } 2 \text { and no } \\
\text { scores less than } 2\end{array}$ \\
\hline Weak & More than three scores of 2 or less \\
\hline Moderate & Scoring combinations other than strong or weak \\
\hline Not available & Two or more gaps in scoring for a particular evaluation question \\
\hline
\end{tabular}

These outcome status assessments, aggregated across all seven country programmes, are shown in Table 1 for each evaluation question. These results identify common trends across WaterAid in relation to specific programme areas where there are consistent strengths or weaknesses. Strong areas of performance are in partnership development, policy advocacy, equity and inclusion; areas requiring attention are: organisational learning; programme structure; and government partner capacity for sustainability.

This analysis identifies aspects of the underlying data that require further exploration in order to understand the issues that have led to the outcome status. These data provide the organisational lessons for prioritising future programme management and development.

For each evaluation question, a qualitative descriptive summary aggregating the findings from each country study was prepared. These were further synthesised into summary statements for each of the Outcome Areas (shown in column 1 of Table 1) by aggregating the summaries from each of the component evaluation questions. These summary findings are described below. The outcome status and its description reveals what worked; the above approach to meta-analysis of the underlying qualitative data reveals how and why it worked - the lessons from experience.

\section{Outcome 1: Partnerships}

\section{Outcome Status: Strong}

Successful partnerships and relationships have been developed and sustained with organisations in the WASH sector both at national level for advocacy and at local level for influencing service delivery. Representatives of national Government, bi-lateral and multi-lateral organizations all noted WaterAid's ability to make a difference to the lives of poor people through this way of working.

\section{Lessons}

Partnerships resulted in substantive visibility and real potential for influence; nationally, WaterAid is one of the few NGOs invited to 'sit at the donor table' as part of joint sector reviews and donor assistance groups. WaterAid's consistent support to the sector, in some cases over a period of 25 years, has brought it into contact with all of the important actors in the sector, many of whom are its partners or collaborators. However not all countries have successfully established themselves in this key role.

Increased focus on relationships with local government e.g. through the development of tripartite arrangements between district government, WaterAid and local partner NGOs, has led to better harmonised approaches and improved programme effectiveness. Improving the geographic focus of WaterAid's national programmes has assisted this. 


\section{Outcome 2: Equity and Inclusion}

\section{Outcome Status: Strong}

WaterAid is addressing the needs of the poorest communities through successfully targeting remote, hard-to-reach and vulnerable communities and marginalised groups. Programmes recognised the need for sensitivity if there is to be a change in behaviours; for example, where patriarchy and religious beliefs may deny equal participation to women. Efforts to encourage equal treatment and access for people with disability and suffering from HIVIAIDS may also provoke disquiet. This continues to be work in progress, with several evaluations being undertaken at the same time as the development of WaterAid's equity and inclusion framework.

\section{Lessons}

WaterAid is recognised as having a strong commitment and emerging expertise in this complex area. Successes have been achieved through analysis of vulnerability criteria, including: marginalisation; disease e.g. cholera-endemic communities; low coverage; and low income. Targeting can be improved through greater use of the excellent waterpoint mapping and other monitoring and evaluation data to ensure vulnerable communities are effectively identified. Some good examples of inclusive design exist e.g. for old and disabled people in both urban and rural programmes, but this needs to more widely adopted.

Adopting a strong geographic focus has again been important when identifying areas of greatest need.

\section{Outcome 3: Programme structure}

\section{Outcome Status: Moderate}

Some programmes respond effectively, recognising the dynamic nature of the national policy and institutional context, and the need to adapt and fit to a changing environment. The picture is mixed: aspects of structuring can be strong but engagement with government programmes can be weak.

\section{Lessons}

Effective programme structures have led to good relationships with local government: e.g. responding to decentralisation by directing WaterAid finance directly through local government structures. Some programmes need to learn from past approaches, structure themselves to have more strategic influence and engage more with those national government programmes that will dominate sector work in the medium term.

Good geographic focus supports complementary outcomes around equity targeting. The rationale for working in chosen districts with all partners is not always clear, resulting in some country programmes that are too diversified both geographically and thematically.

All programmes are at risk of compromise because of internal human resource challenges. A lack of technical staff to adequately support programmatic work, for example through high staff turnover, is a worrying constraint to effective operation. In some cases this relates to the relatively small pool of sector expertise available in-country. Some programmes need to review their management structures to improve their effectiveness and achieve closer integration of practice and policy work.

\section{Outcome 4: Policy advocacy}

\section{Outcome Status: Strong}

WaterAid has been successful in influencing change: e.g. sector 'thinking' about sanitation; adoption of appropriate technologies; and policy adoption at national and state levels. Where 
there has been less success, programmes need to clearly identify their priorities for influencing sector policy and practice.

\section{Lessons}

Close engagement has led to greater effectiveness in influencing attitudes, operations and policy at higher levels of government; this is the first step in strengthening operations through better recognition of key issues. For example in Nigeria, improved WASH policy has been approved and signed into law.

Significant influence has also been leveraged at local level, through building relationships and adopting procedures that are closely aligned to those of local government, for example: harmonising financial flows in Mozambique; and promoting regional legislation to legitimise community-run water management institutions which are gradually being taken up across Ethiopia. Community-level advocacy and service delivery need to go hand-in-hand as people need to see and relate to tangible outcomes. This principle applies from local users' associations through to national Government.

\section{Outcome 5: Organisational Learning}

\section{Outcome Status: Weak}

Most programmes showed insufficient application of learning from the performance and effectiveness of existing programmes to the planning of future work. More analysis of operational experience to enhance links between practice and policy work is needed. There are individual cases of very good practice that offer opportunities for improving intra-country learning exchanges as an effective mechanism for disseminating and sharing good practice. Elsewhere, timely publication and dissemination linked to a strategy for communication and advocacy is absent.

\section{Lessons}

There is scope to improve the effectiveness of WaterAid's programmes through more strategic use of rich operating experience. The availability and use of monitoring data for planning is variable; greater use of these data is the best way to inform future programming.

There are some cases where collaborating organisations report that WaterAid makes very positive contributions to the body of evidence in the sector. In Nigeria there are excellent learning publications that do not shy away from criticising WaterAid's work where justified, bringing out positive and negative experiences in a straightforward way.

\section{Outcome 6: Service delivery and accountability}

\section{Outcome Status: Moderate}

Stakeholders cite WaterAid's track record in service delivery as a benchmark example of the contribution that NGOs can make. Approaches and technologies for water supply are generally suitable but outcomes are more variable in sanitation. The quality of work is generally good, with an awareness of the need for continued vigilance in quality control. Some programmes need to adopt a tighter technical focus and there are concerns about longer term O\&M in relation to some technologies.

\section{Lessons}

Rural water supply: WaterAid generally use tried and tested water supply technologies. In some cases, facilities were recorded as insufficient for the number of people using them. Technical capacity in some areas may be low, e.g. supervision of drilling contractors. 
Rural sanitation and hygiene: variations in approach and success exist between different programmes, ranging from 'total sanitation' with little subsidy, to fully subsidised latrine programmes. Opportunities exist to promote a wider range of sanitation technologies, demonstrating their advantages and disadvantages and to continue to ensure that sanitation and hygiene are a priority.

Urban water supply: there is limited overall success with significant variations between programmes. Development of urban water kiosks in Maputo makes the most of an excellent institutional framework involving user groups with the utility and the regulator. More broadly, renewed efforts are needed to establish stronger partnerships with municipalities, utilities and water users associations.

There is limited operational experience and success with urban sanitation and hygiene. Technology choice can be more complex and unit costs are often high. High levels of subsidy e.g. in the ecological sanitation pilot in Mozambique are not sustainable, resulting in low programme coverage at high cost. There is a need to focus on simple and cheaper alternatives.

\section{Outcome 7: Sustainability}

\section{Outcome Status: Moderate}

Sustainability of services (including continued financing for O\&M and technical support) remain a major constraint in all programmes. However, this is a government responsibility that extends way beyond the remit of a single organisation and the dilemma for WaterAid is how far any external organisation should go in terms of taking over government responsibilities as this itself is not sustainable. WaterAid is doing many of the right things to enable long-term functioning of water supply and sanitation systems and corresponding behaviour changes.

\section{Lessons}

There is severe government understaffing at district level, with under qualified personnel. Local government staff are routinely involved in projects, sometimes through long-term secondments; there are examples of Government picking up and taking forward the capacity that WaterAid has helped establish. Links between communities and District Government need to be strengthened, for example to include the establishment of District-based pump spare parts systems and promoting the role of local community representatives in reporting community WASH status.

Low levels of funding and maintenance contributions characterise the sector with financing and availability of spare parts for water points an on-going problem. WaterAid's proposed pilot work on sustainability in Mozambique offers a good approach to building alliances to work much more with others and look beyond local communities for support.

Overall, there is good long term approach to assessing the effectiveness of WaterAid's partners. New partners are brought on board where required and many of WaterAid's country programmes make use of exchange visits between partners to promote shared learning. . There is a risk of some local partners becoming overly dependent for their existence on WaterAid and more work on developing exit strategies is needed.

\section{Conclusions}

A framework for evaluation of outcomes in water supply, sanitation and hygiene programmes has been developed by WaterAid in response to the difficulties experienced in aggregating the results of earlier evaluations of the work of its 23 operational country programmes. It also proved difficult to assimilate work reported elsewhere as "lessons learned" in order to identify areas of success and hence guide future organisational development. 
The framework combines WaterAid's theory of change, articulated in its organisational strategy and principles, with the OECD DAC Guidelines on evaluation of development projects. A set of 17 standard evaluation questions is developed and used to assess the relevance, effectiveness and sustainability of WaterAid's country programmes. The model applies ordinal scores to each assessment which can be aggregated into an overall assessment of outcome status, rated as: strong; moderate; or weak.

The methodology is successfully applied to seven recent evaluations of WaterAid country programmes. This enables findings on outcome status to be aggregated in a way that has validity at an organisational level, rather than simply at a country programme level. Strong areas of performance are: partnership development; policy advocacy; equity and inclusion. Areas requiring attention are: organisational learning; programme structure; and government partner capacity for sustainability. Analysis of the underlying qualitative data has drawn out specific organisational lessons about what has worked, how it was done and why it was important. These findings will be used to prioritise future programme management and development.

The evaluation questions are clustered into seven broader outcome areas that are likely to be of generic interest to other organisations managing WASH programmes. Whilst this is not offered as a "one size fits all" solution to sector outcome evaluation, it enables over-arching lessons to be drawn with respect to improving both organisational performance and programming for WASH. The framework has potential for wider application to the water and sanitation sector, thereby assisting other organisations to achieve a more coherent set of water and sanitation outcomes and impacts.

\section{References}

Adams, J. (2001) NGOs and Impact Assessment, NGO Policy Briefing Paper No. 3. INTRAC (International NGO Training and Research Centre), Oxford, UK .

Clasen, T.F., Bostoen, K., Schmidt, W-P., Boisson, S., Fung, I.C-H., Jenkins, M.W., Scott, B, Sugden, S. and Cairncross, S. (2010) Interventions to Improve Disposal of Human Excreta for Preventing Diarrhoea. Cochrane Database of Systematic Reviews, Issue 6, Art. No.. CD007180. DOI: 10.1002/14651858.CD007180.pub2

Denscombe, M (2010) The Good Research Guide for small scale social research projects, Fourth Edition, Open University Press, Maidenhead, UK

Development Assistance Committee (1991) Principles for Evaluation of Development Assistance. OECD, Paris. [online] http://www.oecd.org/dataoecd/21/32/41029845.pdf [accessed 18 October 2011].

Development Assistance Committee (undated) DAC Criteria for Evaluating Development Assistance, Factsheet. OECD, Paris. [online] http://www.oecd.org/dataoecd/15/21/39119068.pdf [accessed 18 October 2011].

GLAAS (2010) UN-Water Global Annual Assessment of Sanitation and Drinking-Water. WHO, Geneva, Switzerland.

Higgins, J.P.T. and Green, S. (editors) (2011) Cochrane Handbook for Systematic Reviews of Interventions Version 5.1.0 [updated March 2011]. The Cochrane Collaboration, 2011.[online] www.cochrane-handbook.org [accessed 18 October 2011].

Hutton, G. and Haller, L. (2004) Evaluation of the Costs and Benefits of Water and Sanitation Improvements at the Global Level. Water, Sanitation and Health Protection of the Human Environment, WHO, Geneva, Switzerland. 
IRC (2011) Lessons Learnt from Sanitation and Hygiene: Practitioners' Workshops 2007 - 2011. IRC International Water and Sanitation Centre, Delft, The Netherlands.

JMP (2010) Progress on sanitation and drinking water: 2010 update. WHO/UNICEF Joint Monitoring Programme (JMP) for Water Supply and Sanitation, WHO, Geneva, Switzerland.

Leeuw, F. and Vaessen, J. (2009) Impact Evaluations and Development: NONIE Guidance on Impact Evaluation. NONIE-The Network of Networks on Impact Evaluation, Independent Evaluation Group, Washington DC. [online] http://siteresources.worldbank.org/EXTOED/Resources/nonie guidance.pdf [accessed 18 October 2011].

Malhotra, N. and Birks, D (2000) Marketing Research: An Applied Approach, Prentice-Hall Inc, Harlow, UK

Saywell, D. and Hunt, C. (1999) Sanitation Programmes Revisited, WELL Task 161, p13. WEDC, Loughborough University, UK.

Waddington, H., Snilstveit, B., White, H. And Fewtrell, L. (2009) Water, sanitation and hygiene interventions to combat childhood diarrhoea in developing countries, The International Initiative for Impact Evaluation (3ie), New Delhi India, London UK and Washington DC USA

WaterAid (2009) WaterAid's Global Strategy 2009-2015. WaterAid, London.

WaterAid (2010) External Country Programme Evaluations. WaterAid, London.

WELL (1998) DFID Guidance Manual on Water Supply and Sanitation Programmes. WEDC, Loughborough University, UK.

WELL (2005) Will it cost the earth? An overview of cost estimates for achieving the water and sanitation targets of the Millennium Development Goals, WELL Briefing Note 9. WEDC, Loughborough University, UK.

WordPress (2011) WASH Lessons Learned [online] http://washlessons.wordpress.com/ [accessed 31 August 2011] 
Table 1. Summary of Country Programme Evaluation Findings

\begin{tabular}{|c|c|c|c|c|c|c|c|c|c|c|}
\hline $\begin{array}{c}\text { Outcome area } \\
(1)\end{array}$ & & Evaluation Questions & $\begin{array}{c}\text { Outcome } \\
\text { Status }\end{array}$ & Malawi & Zambia & Ethiopia & Nigeria & Ghana & Uganda & Moz'bque \\
\hline $\begin{array}{l}\text { Programme } \\
\text { structure }\end{array}$ & $\mathrm{R} 1$ & $\begin{array}{l}\text { WaterAid's analysis of (and response to) the national } \\
\text { WASH sector, relevant policies and identified sector } \\
\text { blockages }\end{array}$ & Moderate & & 4 & 2 & 3 & 3 & 3 & 3 \\
\hline $\begin{array}{l}\text { Programme } \\
\text { structure }\end{array}$ & $\mathrm{R} 2$ & $\begin{array}{l}\text { How the country programme have structured themselves } \\
\text { in relation to identified blockages }\end{array}$ & Weak & 2 & 3 & 2 & 2 & 2 & 2 & 2 \\
\hline $\begin{array}{l}\text { Programme } \\
\text { structure }\end{array}$ & R3 & $\begin{array}{l}\text { The rationale for targeting programmes of work in specific } \\
\text { districts, or particular issues }\end{array}$ & Moderate & 3 & 3 & 2 & 3 & 4 & 2 & 4 \\
\hline Service delivery & R4 & $\begin{array}{l}\text { The suitability of supported technologies and approaches } \\
\text { used in service delivery work }\end{array}$ & Moderate & 3 & 3 & 3 & 3 & 3 & & 3 \\
\hline $\begin{array}{l}\text { Equity \& } \\
\text { inclusion }\end{array}$ & R5 & $\begin{array}{l}\text { The approaches used to engage and involve marginalised } \\
\text { and excluded groups }\end{array}$ & Moderate & 2 & 3 & 3 & 3 & & 3 & 3 \\
\hline Partnerships & R6 & $\begin{array}{l}\text { The appropriateness of partnerships and relationships } \\
\text { with other organisations involved in the WASH sector }\end{array}$ & Strong & 3 & 4 & 4 & 2 & 4 & 3 & 3 \\
\hline Policy advocacy & $\mathrm{R} 7$ & $\begin{array}{l}\text { Whether policy advocacy work has been directed towards } \\
\text { high priority areas, or for strategically opportunistic } \\
\text { purposes. }\end{array}$ & Moderate & 4 & 3 & 2 & 3 & 4 & 2 & 2 \\
\hline $\begin{array}{l}\text { Equity \& } \\
\text { inclusion }\end{array}$ & E1 & $\begin{array}{l}\text { How effectively are partners promoting (and securing) } \\
\text { rights and access to WASH }\end{array}$ & Moderate & 3 & 4 & 3 & 3 & 3 & 3 & 3 \\
\hline $\begin{array}{l}\text { Equity \& } \\
\text { inclusion }\end{array}$ & E2 & $\begin{array}{l}\text { How have the most vulnerable and marginalised } \\
\text { communities (and people within those communities) been } \\
\text { identified, and how have their needs been met }\end{array}$ & Strong & 2 & 4 & 4 & 4 & & 3 & 3 \\
\hline Service delivery & E3 & What is the quality of supported service delivery work & Moderate & 4 & 3 & 2 & 3 & 3 & & 3 \\
\hline Partnerships & E4 & $\begin{array}{l}\text { How effective has WA been in fostering productive } \\
\text { relationships between service providers and local } \\
\text { governments }\end{array}$ & Moderate & 3 & & 3 & 3 & 3 & 3 & 3 \\
\hline Partnerships & E5 & $\begin{array}{l}\text { How effective (or otherwise) have WA's capacity building } \\
\text { initiatives been with partners and LGs (specifically in } \\
\text { relation to programme principles) }\end{array}$ & Moderate & 3 & 2 & 3 & 2 & & 2 & 3 \\
\hline Policy advocacy & E6 & $\begin{array}{l}\text { Have WA supported initiatives contributed to changing the } \\
\text { policy and practice work of others in the WASH sector }\end{array}$ & Strong & 3 & 4 & 3 & 4 & 4 & & 2 \\
\hline $\begin{array}{l}\text { Organisational } \\
\text { learning }\end{array}$ & E7 & $\begin{array}{l}\text { Have findings from past experiences, reports, studies or } \\
\text { other events informed future work and led to improved } \\
\text { effectiveness }\end{array}$ & Weak & 2 & 2 & 2 & 2 & 2 & 2 & 2 \\
\hline Sustainability & E8 & $\begin{array}{l}\text { What is the evidence and / or plausibility of WASH } \\
\text { services functioning (and being used) over the long term }\end{array}$ & Moderate & 3 & 3 & 2 & 3 & 3 & 3 & 3 \\
\hline Sustainability & E9 & $\begin{array}{l}\text { Do national and local governments have the capacity and } \\
\text { resources to manage and maintain services in the future }\end{array}$ & Weak & 1 & 1 & 3 & 2 & 1 & 2 & 1 \\
\hline Sustainability & E10 & Do WaterAid's relationships with partners consider the & $\mathrm{N} / \mathrm{A}$ & 3 & 3 & & & 2 & 3 & 3 \\
\hline
\end{tabular}


Notes.

1. Blank cells indicate insufficient information to assign a score

2. Outcome Areas (column 1) are labelled to indicate the their broad area of general application

3. Evaluation Questions are concerned with relevance $(R)$ and effectiveness $(E)$ as indicated in column 2 